Relations industrielles

Industrial Relations

\title{
La sécurité sociale en Grande Bretagne
}

\section{Edgar Guay}

Volume 5, numéro 4, janvier 1950

URI : https://id.erudit.org/iderudit/1023304ar

DOI : https://doi.org/10.7202/1023304ar

Aller au sommaire du numéro

Éditeur(s)

Département des relations industrielles de l'Université Laval

ISSN

0034-379X (imprimé)

1703-8138 (numérique)

Découvrir la revue

Citer cet article

Guay, E. (1950). La sécurité sociale en Grande Bretagne. Relations industrielles / Industrial Relations, 5(4), 31-32. https://doi.org/10.7202/1023304ar

Tous droits réservés @ Département des relations industrielles de l’Université Laval, 1950
Ce document est protégé par la loi sur le droit d'auteur. L'utilisation des services d'Érudit (y compris la reproduction) est assujettie à sa politique d'utilisation que vous pouvez consulter en ligne.

https://apropos.erudit.org/fr/usagers/politique-dutilisation/ 


\section{Bulletin des relations industrielles}

Volume 5, numéro 4

QUÉBEC

janvier 1950

\section{LA SÉCURITÉ SOCIALE EN GRANDE BRETAGNE}

\section{Edgar Guay}

Le Britannique, homme d'action, a traduit ce concept de « sécurité sociale» en un vaste réseau de services sociaux synchronisés avec un système d'assurances nationales couvrant tous les risques communs «from womb to tomb», pour employer l'expression usuelle.

Bien que le plan à la base de toute la législation instituant les services et les intégrant d'une façon rationnelle fût l'oeuvre d'un comité interdépartemental, la personnalité de son président, Lord Beveridge, était si grande que son nom servit à désigner ce document depuis sa publication en 1942. Ce plan est la résultante de la poussée de deux problèmes, l'un d'ordre administratif, l'autre du domaine social.

En 1942, le réseau administratif des services sociaux publics était semblable à une jungle où le citoyen avait peine à se retrouver, malgré l'aide des Citizen's Advice Bureaux et des services de renseignement des Trade Unions. Sept ministères, environ deux cents autorités locales, des centaines de sociétés mutuelles et compagnies d'assurance entrelaçaient leur activité dans ce domaine.

Le problème social classique, la pauvreté, avait été remis à l'ordre du jour par une série d'enquêtes scientifiques menées dans les grands centres industriels. En plus d'exposer l'étendue du mal et ses causes, ces enquêtes révélaient la suffisance des ressources de la communauté pour y mettre fin. Une simple redistribution du revenu ferait disparaître la carence de ressources financières des économiquement faibles. Cette redistribution de revenu serait opérée par un système d'assurances sociales et de services gratuits connexes auquels chacun pourrait recourir de droit et sans enquête sur l'état de ses ressources.

Une politique d'embauchage intégral devrait aussi être élaborée; l'emploi, en effet, en assurant le revenu, est la pierre angulaire de la sécurité sociale. Beveridge reconnaissait les limites d'un tel système en affirmant que c'était là un minimum sur lequel les citoyens pourraient construire librement.

Les recommandations du Plan Beveridge ont été traduites par une série de mesures légales et la mise sur pied de quatre classes de services interdépendants: 1 . les services du maintien du revenu, 2. les services d'embauchage et de réhabilitation, 3. les services sanitaires, 4. les services de bien-être des autorités locales.

La première classe est constituée par les assurances sociales auxquelles contribuent tous les citoyens, selon leur status, de même que l'Etat et les employeurs. Les allocations familiales, qui aident à maintenir à un niveau raisonnable le revenu des familles croissantes, font aussi partie de cette catégorie. Il en est de même de l'Assistance nationale, qui soutient la continuité du revenu en cas d'urgence et de cessation du droit aux assurances sociales.

La population dans son ensemble est protégée par plus d'un millier de bureaux locaux d'assurances sociales et trois cents bureaux d'assistance conseillés par une myriade de comités où sont représentées toutes les classes de la société. Le Ministère des assurances nationales veille à la bonne administration de ce secteur.

Un réseau aussi dense de bureaux de placement facilite le passage d'un emploi à l'autre. Ces bureaux entretiennent des relations étroites avec les centres de réadaptation professionnelle et de réhabilitation physique afin que tous les citoyens aient l'avantage d'obtenir un emploi rémunérateur. Ce groupe de services est confié au Ministère du travail.

Le Ministère de la santé, pour sa part, dirige les services sanitaires auxquels tous les citoyens 
ont accès gratuitement sans égard à leur status vis-à-vis l'assurance nationale. Hôpitaux, cliniques, spécialistes, médecins, dentistes, produits pharmaceutiques, services ophtalmiques, tout est mis à la disposition du plus humble des citoyens et même de l'étranger en visite. En pratique, ce secteur est entièrement financé par l'Echiquier au moyen des ressources prélevées des contribuables par voie de taxation.

Les municipalités des villes et des comtés mettent aussi à la disposition de la population nécessiteuse des institutions de bien-être: maisons pour les vieillards pensionnés de l'Etat, centres de puériculture, services d'aides familiales et autres services annexes destinés à compléter, sur le plan local, les grands services nationaux.

Le fardeau du coût global de tous ces services peut être réalisé quand on se souvient qu'avant la guerre, le gouvernement recueillait environ $20 \%$ du revenu national, alors qu'aujourd'hui il en recueille $37 \%$. Avant la guerre, environ $8 \%$ des dépenses gouvernementales étaient effectuées dans le secteur des services sociaux; à l'heure actuelle, ce pourcentage est monté à $16 \%$, ce qui correspond à une augmentation de près de $100 \%$ des dépenses dans ce domaine.

Cette politique de redistribution de revenu a eu comme effet de niveler la richesse individuelle. En 1939, plus de 11,000 personnes en Angleterre jouissaient d'un revenu annuel supérieur à 5,000 livres sterling après les déductions usuelles; aujourd'hui, le nombre de ces choyés de la fortune est tombé aux environs de 250 . Le système de redistribution a été si efficace que Sir Stafford Cripps, dans le discours du budget en avril dernier, a laissé clairement entendre qu'on ne pouvait s'engager plus avant dans cette redistribution du revenu sans infirmer l'économie nationale.

Le coût de l'administration est aussi couvert par des contributions tri-partites. Pour l'assurance, par exemple, l'employé paie hebdomadairement quatre chelins et deux deniers, son employeur, quatre chelins et 11 deniers, ce qui fait un total de 9 chelins et 1 denier ( $\$ 1.82$ avant la dévaluation récente). Le gouvernement fournit un autre montant global équivalant à $42 \%$ du coût total des assurances sociales. Les autres services de la sécurité sociale sont financés à même la taxation.

Ce plan qui englobe la totalité de la population a subi pour une quinzaine de mois l'épreuve de la mise en pratique. Des résultats généraux ont été obtenus, par exemple: 95\% des Britanniques ont consulté les services sanitaires, le coût d'administration de ce secteur a dépassé de $100 \%$ les estimés, malgré la participation bénévole de 10,000 citoyens sur les comités d'administration. En janvier 1949, l'Assistance nationale comptait sur ses listes plus d'un million de clients dont les trois quarts recevaient des prestations de l'assurance sociale. Beveridge avait prévu un rôle d'arrière-plan pour cet organisme, mais il semble prendre de l'ampleur à cause de l'incapacité relative de l'Assurance nationale de donner aux Britanniques le minimum de subsistance prévuı.

Aucun des grands partis politiques anglais ne s'oppose à ces mesures de sécurité sociale. Le parti libéral se targue d'être à l'origine du mouvement, avec la législation de Lloyd George en 1911, et d'être intervenu en 1942 par son plus illustre représentant, Lord Beveridge. Il favorise une politique d'administration suivant une ligne ne déviant ni vers la gauche ni vers la droite. Mais ce parti, malgré les efforts accomplis pour le renflouer, n'a pratiquement plus d'influence.

Le parti conservateur réclame aussi son droit de paternité sur la majeure partie de la législation sociale. Churchill et Sir John Anderson ont travaillé à l'édification de la structure des services sociaux publics depuis le début de leur carrière politique. Le rapport Beveridge fut rédigé à la demande du gouvernement de coalition dirigé par Churchill. Le parti conservateur, à l'heure présente, désire maintenir tous les services sociaux mais souhaite une meilleure administration en attirant l'attention de l'électorat sur leur coût élevé. Il pose à l'électorat la question: "Combien l'Angleterre peut-elle supporter de services?»

A cette question les travaillistes, avec Aneurin Bevan, répondent: «Autant qu'en requiert le besoin ». On peut se demander quelle sera, dans la pratique, la conduite du parti au pouvoir, car Sir Stafford Cripps, dans les derniers estimés budgétaires, recommande, par mesure d'économie, l'arrêt de la construction des centres sanitaires par tout le pays. De plus, un contribution de $\mathbf{0 . 1 4}$ sera aussi exigée du patient pour chaque ordonnance médicale qu'il remettra au pharmacien. On espère ainsi diminuer quelque peu le nombre effarant des $187,000,000$ d'ordonnances médicales distribuées en 1948-49.

Les données des statistiques vitales pour l'année fiscale 1948-49 et les répercussions économiques de la dévaluation récente seront probablement les facteurs qui influenceront le plus l'avenir immédiat de la sécurité sociale en Grande Bretagne. 